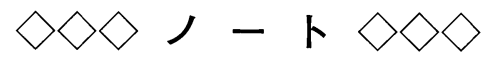

\title{
A Model for Describing the Desorption Processes of $\beta$-Lactogloblin Fouled on Stainless Steel Surface during Caustic and Enzymatic Cleanings
}

\author{
Shuji ADACHI $^{1, \dagger}$, Kazuhiro NAKANISHI ${ }^{2}$, and Ryuichi MATSUNO ${ }^{1}$ \\ ${ }^{1}$ Division of Food Science and Biotechnology, Graduate School of Agriculture, Kyoto University, \\ Sakyo-ku, Kyoto 606-8502, Japan \\ ${ }^{2}$ Department of Bioscience and Biotechnology, Faculty of Engineering, Okayama University, \\ 3-1-1 Tsushima-naka, Okayama 700-8530, Japan
}

\begin{abstract}
The desorption processes of $\beta$-lactoglublin fouled on a stainless steel surface during caustic and enzymatic cleanings were analyzed using the model in which the free energy of activation for the rate constant of the first-order kinetics was assumed to obey a Gaussian distribution. The model well expressed both processes. This would indicate that the $\beta$-lactogloblin molecules exist in various states and interact with the surface in various strengths corresponding to the states. The rate constant corresponding to the mean value of the free energy of activation was greater at the higher detergent concentrations for both the caustic and enzymatic cleanings.
\end{abstract}

Key words: cleaning, fouling, $\beta$-lactogloblin, free energy of activation

\section{Introduction}

The cleaning of fouled surfaces of food processing equipment is an essential process to provide the sanitary condition of products although it is a non-productive one. Caustic solution is mainly used as a detergent in the food industries. The use of enzymes such as proteases would also be effective for the cleaning of proteineous deposits. To reasonably design the cleaning processes, the rate process of the desorption of a fouled deposit from the surface should be well understood. We have reported the desorption processes of $\beta$-lactoglobline, which is one of the main constituents of the fouling deposits in milk processing [1] from a stainless steel surface during caustic and enzymatic cleanings [2]. The first half of each desorption process obeyed first-order kinetics, but the latter half did not. Therefore, a kinetic model that can describe the entire desorption process is required.

Bourne and Jennings [3] showed that two species of tristearin were present on stainless steel surface and that each of them was removed from the surface independently and simultaneously by a first-order process. Ellipsoidal protein molecules adhere to the surface in the end-on and side-on modes [4] and change their states on the surface due to denaturation. Therefore, it seems that $\beta$-lactobloblin molecules exist in various states on the surface and

(Received 1 May 2002: accepted 24 May 2002)

Fax: 075-753-6285, E-mail: adachi@kais.kyoto-u.ac.jp their desorption processes depend on these states.

We proposed a kinetic model in which the free energy of activation for the rate constant of the first-order kinetics was assumed to obey a Gaussian distribution to describe the heterogeneity in the denaturation process of the $\alpha$ chymotripsin covalently bound to a water-insoluble support [5]. The model was also applicable to the alkaline nitrobenzene oxidation of lignin [6] and the oxidation of linoleic acid encapsulated with a polysaccharide [7]. In the latter case, the rate equation was the autocatalytic type.

In this study, the kinetic model was applied to our previous data on the desorption processes of $\beta$-lactogloblin fouled on a stainless steel surface during caustic and enzymatic cleanings [2].

\section{Model}

The model is briefly described. There would be many $\beta-$ lactobloblin species on a stainless steel surface. Each of them is assumed to desorb independently and simultaneously according to first-order kinetics. Diversity in the desorbability would be reflected in the rate constant of the first-order kinetics. Based on Eyring's theory of the absolute reaction rate, the rate constant $k$ is related to the free energy of activation, $\Delta G^{\ddagger}$, by Eq. (1).

$$
k=\left(\frac{\kappa_{\mathrm{B}} T}{h}\right) \exp \left(-\frac{\Delta G^{\ddagger}}{R T}\right)
$$

where $h$ is Planck's constant, $\kappa_{\mathrm{B}}$ is Boltzmann's constant, $R$ is the gas constant, and $T$ is the absolute temperature. Let 
us assume that the $\Delta G^{\neq}$value obeys a Gaussian distribution with the standard deviation $\sigma$ and mean value $\Delta \bar{G}^{\neq}$

$$
\begin{aligned}
f\left(\Delta G^{\neq}\right) & =\frac{1}{\sqrt{2 \pi \sigma}} \exp \left[-\frac{\left(\Delta G^{\neq}-\Delta \bar{G}^{\neq}\right)^{2}}{2 \sigma^{2}}\right] \\
& =\frac{1}{\sqrt{2 \pi \sigma}}\left[-\frac{R^{2} T^{2}(\ln k-\ln \bar{k})^{2}}{2 \sigma^{2}}\right]
\end{aligned}
$$

where $\bar{k}$ is the rate constant corresponding to $\Delta \bar{G}^{\neq}$. The fraction of a specie $i$ remaining on the surface at any time $t$, $y_{i}$, can be expressed by Eq. (3) from the integrated form of the first-order kinetics.

$$
y_{i}=\exp \left(-k_{i} t\right)
$$

where $k_{i}$ is the rate constant for desorption of the specie $i$. From these equations, the overall fraction of $\beta-$ lactogloblin remaining on the surface at the time $t, y$, is given by Eq. (4).

$$
y=\frac{R T}{\sqrt{2 \pi \sigma}} \int_{-\infty}^{\infty} \exp \left[-\frac{R^{2} T^{2}(\ln k-\ln \bar{k})^{2}}{2 \sigma^{2}}\right] \exp (-k t) d(\ln k)
$$

\section{Results and Discussion}

\section{1 Applicability of the model to cleaning processes}

Figures 1(a) and (b) show the desorption processes of $\beta$-lactogloblin from a stainless steel (SUS316) surface during caustic and enzymatic cleanings, respectively, at $50^{\circ} \mathrm{C}$ [2]. For the enzymatic cleaning, Protin AC10 was used as the detergent. The above-mentioned model was applied to analyze the processes. Although the processes were observed in a flow system using a column packed with stainless steel particles fouled with the protein, Eq. (4) for a batch desorption process was used in the analysis because the residence time was very short compared with the cleaning period in every case. Equation (4) has two unknown parameters, $\bar{k}$ and $\sigma$. A set of $\bar{k}$ and $\sigma$ values that fit the experimental $y$ values was determined by the trialand-error method to minimize the sum of the residual squares between the calculated and experimental results for each process. The curves are the ones calculated using the best-fitted $\bar{k}$ and $\sigma$ values. The curves well expressed the experimental results for both the caustic and enzymatic cleanings at any detergent concentration. The applicability of the model to the processes indicates that $\beta$ lactogloblin molecules interact with the surface in various strengths.

The $y$ values leveled off in some processes. The model could not describe the leveling off although the desorption rate calculated by the model at prolonged cleaning time was very slow. This would be a limitation of the model.

\section{2 Dependences of the parameters on the detergent concentraton}

The previously determined $\bar{k}$ and $\sigma(\sigma / R T)$ values are plotted versus the detergent concentrations in Fig. 2. The $\bar{k}$ value increased with the increasing sodium hydroxide concentration and reached a plateau at the higher caustic cleaner concentrations. The $\sigma / R T$ values were about 1 except at $0.01 \mathrm{~mol} / \mathrm{L}$ sodium hydroxide. Fryer et al. stated in their review [8] that there was an optimum concentration for a caustic detergent and that the cleaning efficiency decreased at the higher concentrations. Such a phenomenon was not observed in the range

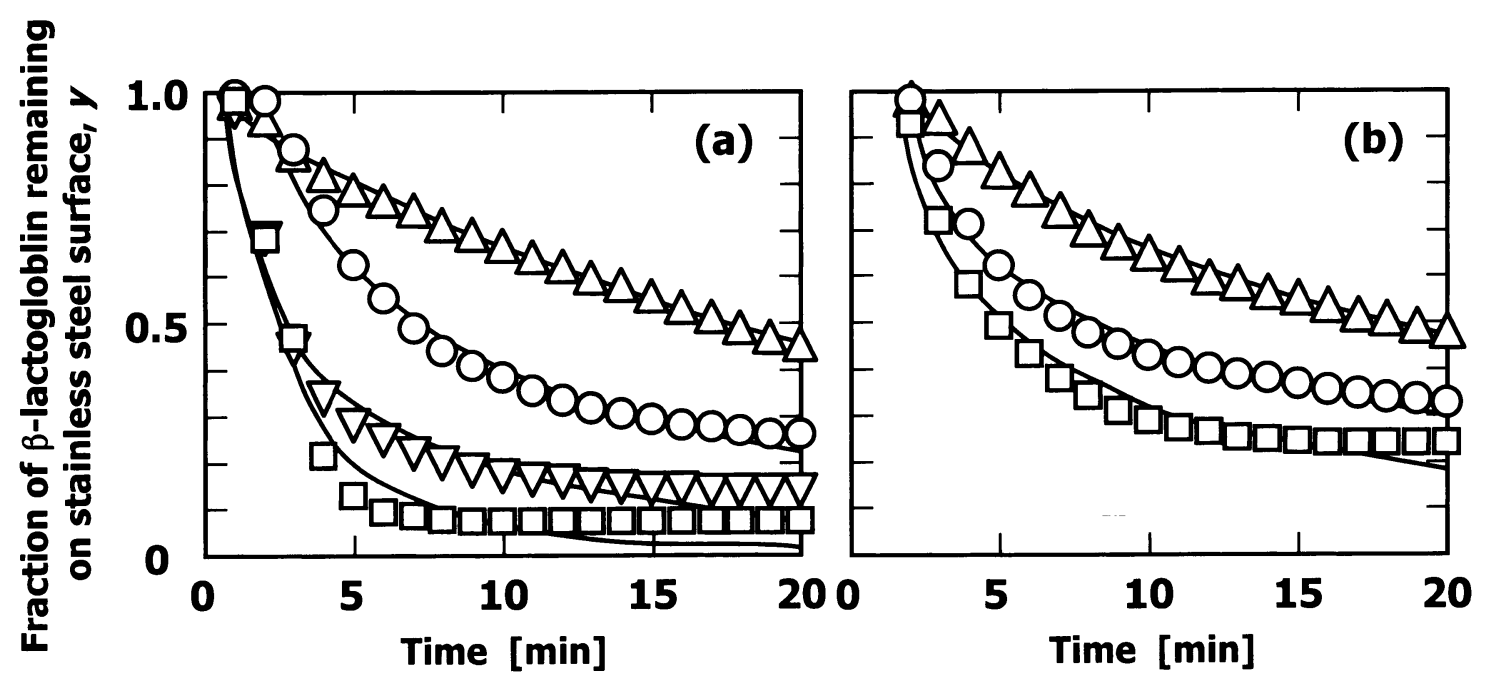

Fig. 1 Desorption processes of $\beta$-lactogloblin fouled on stainless steel surface during (a) caustic and (b) enzymatic cleanings at $50^{\circ} \mathrm{C}$. (a) Sodium hydroxide concentrations: $(\triangle) 0.01,(\bigcirc) 0.02,(\nabla) 0.1$ and $(\square) 0.5 \mathrm{~mol} / \mathrm{L}$. (b) Enzyme, Protin AC10, concentrations: $(\triangle) 1.29$, $\bigcirc) 13.9$ and $(\square) 32.3 \mathrm{mg} / \mathrm{L}$. 


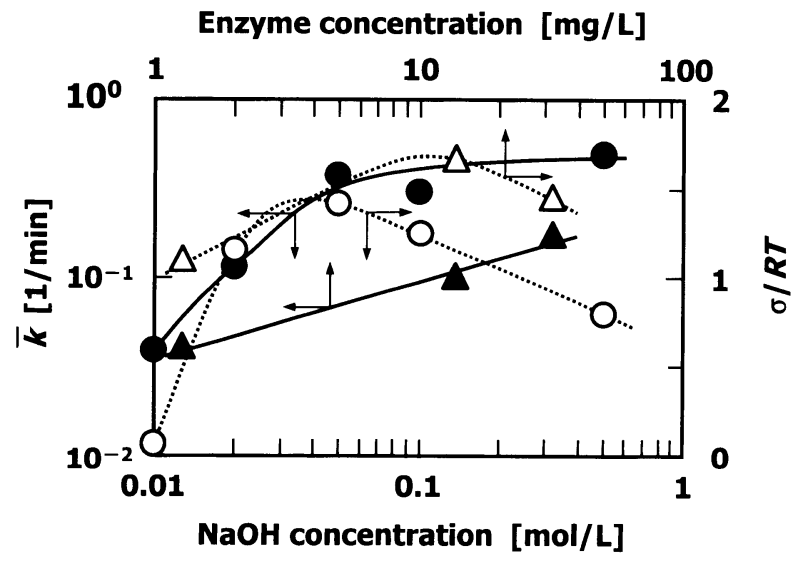

Fig. 2 Dependencies of the $\bar{k}$ and $\sigma / R T$ values (closed and open symbols, respectively) on the detergent concentration for the caustic (circles) and enzymatic (triangles) cleanings of $\beta-$ lactogloblin fouled on a stainless steel surface using sodium hydroxide and Protin AC10 as detergents.

of the concentrations tested in this study.

During the enzymatic cleaning, the $\bar{k}$ value increased with the increasing protease concentration. The $\sigma / R T$ values in this enzymatic cleaning were slightly greater than those in the caustic cleaning.

As mentioned above, the model could well express the characteristics of the desorption processes of $\beta$ lactogloblin from a stainless steel surface during caustic and enzymatic cleanings. A model in which the rate constant $k$ itself was assumed to obey a Gaussian distribution could not express these characteristics. Fouling deposits found in the food industries are composed of multi-components. The distribution of the components in the deposits is not homogeneous, and some components interact with each other [9]. The applicability of the model to the cleaning of such deposits, and its limitation should be further examined.

\section{Acknowledgment}

This study was conducted as a part of the study supported by the Japan Society for the Promotion of Science as a Grant-in-Aid for Scientific Research (C) (Project No. 13895014).

\section{References}

1) J. P. Tissier, M. Lalande, G. Corrieu; A study of milk deposit on heat exchanger surface during UHT treatment. In "Engineering and Food" ed. by B. M. McKenna, Elsevier Applied Science Publishers, Essex, UK, 1984, pp. 49-58.

2) A. Nagata, T. Sakiyama, H. Itoh, T. Toyomasu, E. Enomoto, T. Nagai, T. Saeki, K. Nakanishi; Comparative study on caustic and enzymatic cleanings of stainless steel surface fouled with $\beta$-lactogloblin. Biosci. Biotechnol. Biochem., 59, 2277-2281 (1995).

3) M. C. Bourne, W. G. Jennings; Existence of two soil species in detergency investigations. Nature, 197, 10031004 (1963).

4) K. Imamura, E. Ikeda, T. Nagayasu, T. Sakiyama, K. Nakanishi; Adsorption behavior of methylene blue and its congeners on a stainless steel surface. J. Colloid Interface Sci., 245, 50-57 (2002).

5) Y. Kawamura, K. Nakanishi, R. Matsuno, T. Kamikubo; Stability of immobilized $\alpha$-chymotrypsin. Biotechnol. Bioeng., 23, 1219-1236 (1981).

6) S. Adachi, M. Tanimoto, M. Tanaka, R. Matsuno; Kinetics of the alkaline nitrobenzene oxidation of lignin in rice straw. Chem. Eng. J., 49, B17-B21 (1992).

7) E. Ishido, K. Hakamata, Y. Minemoto, S. Adachi, R. Matsuno; Oxidation process of linoleic acid encapsulated with a polysaccharide by spray-drying. Food Sci. Technol. Res., 8, 85-88 (2002).

8) P. J. Fryer, M. T. Belmar-Beiny, P. J. R. Schreier; Fouling and cleaning: mechanisms and models. In "Developments in Food Engineering” ed. by T. Yano, R. Matsuno and K. Nakamura, Blackie Academic \& Professional, Glasgow, UK. 1994, pp. 24-29.

9) K. Nakanishi, K. Imamura, T. Sakiyama; Cleaning and adhesion of proteineous soils in food and biochemical processes (in Japanese), Seibutso-kogaku Kaishi, 77, 241-245 (1999). 